\title{
Coupling Efficiency Measurements for Long-pulsed Solid Sodium Laser Based on Measured Sodium Profile Data
}

Kai Jin ${ }^{1,2,3}$, Kai Wei ${ }^{1,2, *}$, Shiyong Xie ${ }^{4}$, Yong Bo ${ }^{4}$, Junwei Zuo ${ }^{4}$, Pengyuan Wang ${ }^{3,4}$, Lu Feng ${ }^{5}$, Xianghui Xue ${ }^{6}$, Min Li ${ }^{1,2}$, Xuewu Cheng ${ }^{7}$, Chaolong Cui ${ }^{8}$, Yu Shen ${ }^{4}$, Qi Bian ${ }^{4}, \mathrm{Ji} \mathrm{Yao}^{4}$, Angel Otarola $^{9}$, Xiaolin Dai ${ }^{1,2,3}$, Qinjun Peng ${ }^{4}$, Changhui Rao ${ }^{1,2}$, Zuyan $\mathrm{Xu}^{4}$, Yudong Zhang ${ }^{1,2}$

1. Institute of Optics and Electronics, Chinese Academy of Science, Chengdu, China

2. The Key Laboratory on Adaptive Optics, Chinese Academy of Science, Chengdu, China

3. University of Chinese Academy of Sciences, Beijing, China

4. Technical Institute of Physics and Chemistry, Chinese Academy of Science, Beijing, China

5. National Observatory of China, Chinese Academy of Science, Beijing, China

6. University of Science and Technology of China, Hefei, China

7. Wuhan Institute of Physics and Mathematics, Chinese Academy of Science, Wuhan, China

8. Anhui Institute of Optics and Fine Mechanics, Chinese Academy of Science, Hefei, China

9. Thirty Meter Telescope Corporation, California, USA

\begin{abstract}
In 2013, a serial sky test has been held on 1.8 meter telescope in Yunnan observation site after 2011-2012 Laser guide star photon return test. In this test, the long-pulsed sodium laser and the launch telescope have been upgraded, a smaller and brighter beacon has been observed. During the test, a sodium column density lidar and atmospheric coherence length measurement equipment were working at the same time. The coupling efficiency test result with the sky test layout, data processing, sodium beacon spot size analysis, sodium profile data will be presented in this paper.
\end{abstract}

Key Words: Coupling efficiency, Laser guide star, Sky-test, Sodium column density

\section{INTRODUCTION}

Adaptive optics (AO) with sodium Laser Guide Star (LGS) techniques are used, or planned to be used in several observatories, to increase the sky-coverage of the AO system [1-8]. One key of these techniques is using an appropriate laser to generate a sodium beacon bright enough for the wavefront sensor (WFS).

Laser Physics Research Center of Technical Institute of Physics and Chemistry (TIPC), Chinese Academy of Science (CAS) has been studied on the long pulsed sodium laser since 2008. In 2011, on-sky test of the first prototype laser was held by K. Wei [9]. The average power of the laser was $20 \mathrm{~W}$ and a sodium beacon of $8.7 \mathrm{~V}$-magnitude was successfully obtained. And also, modeling of the coupling efficiency of the TIPC laser was given by S. M. Rochester [10]. In the next two years, TIPC team has upgraded the laser. The maximum output power is increased and the stability of the linewidth is improved. The TIPC laser has chosen to be the potential laser for the Thirty Meter Telescope (TMT) project.

On-sky tests for the new TIPC laser have been held at 2013 test. During the sky test, an artificial sodium beacon has been successfully generated. Measurements of the photon returns and coupling efficiency of the laser have been accomplished. The brightness of the sodium beacon is about $8.2 \mathrm{M}$ in V-Band. An enhancement of $60 \%$ was observed when the polarization of the laser changes from linear to circular. Average coupling efficiency of 160 photons $/ \mathrm{s} / \mathrm{W} /($ atoms $/ \mathrm{m} 2)$ was observed at 7 arcsec spot size while using circular polarized light. 


\section{EXPERIMENT}

\subsection{Layout of the Experiment}

The basic layout of our experiment is same as that in 2011 test, as showed in Figure 1. The beam size is about $10 \mathrm{~mm}$ before entering into the laser launch telescope (LLT). The size comes out from the LLT is about $200 \mathrm{~mm}$ if the $200 \mathrm{~mm}$ LLT is used (20x expander) or $240 \mathrm{~mm}$ if the $300 \mathrm{~mm}$ LLT is used (24x expander). During the test, the laser was only launched to the sky in zenith. The 1.8 meter telescope is used as the receiving telescope, and a CCD is mounted at the Nasmyth focal plane to calibrating the brightness of the sodium beacon. The distance between the LLT and 1.8 meter receiving telescope is about $5.2 \mathrm{~m}$.

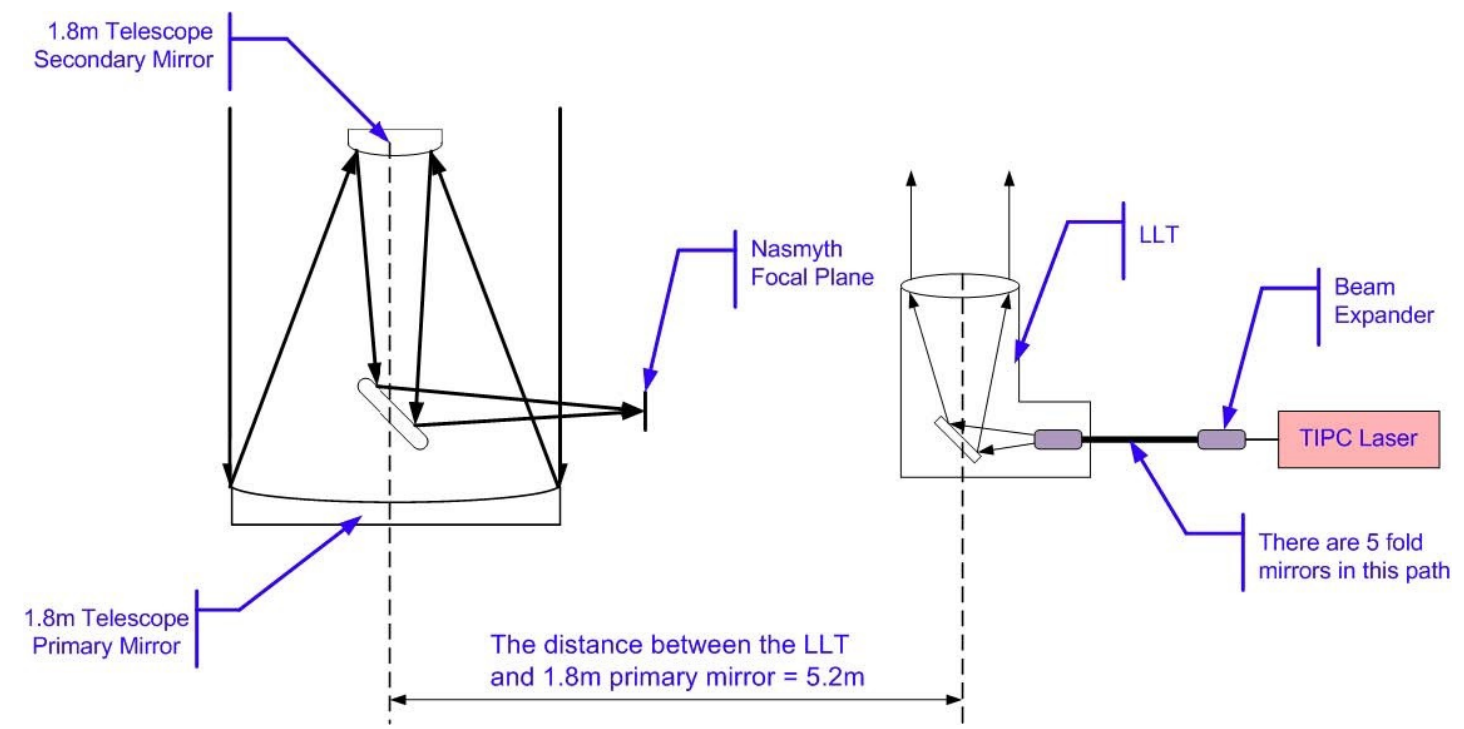

Figure 1: Schematic diagram of the experiment of the TIPC laser on-sky test

After the sky test in 2011, TIPC has upgraded their sodium laser. The output power, wavelength stability, and the beam jitter are all improved. Main parameters of the laser are summarized in Table 1.

Table 1. Main parameters of the TIPC sodium laser

\begin{tabular}{|l|l|}
\hline Maximum Output Power & $32 \mathrm{~W} @ 500 \mathrm{~Hz}, 26 \mathrm{~W} @ 600 \mathrm{~Hz}$ \\
\hline Wavelength & $\begin{array}{l}\text { Center of sodium D2a line, } \\
\text { with ability to tune on/off }\end{array}$ \\
\hline Linewidth & $\sim 0.6 \mathrm{GHz}$ \\
\hline Beam Quality & $\mathrm{M}^{2} \sim 1.5$ \\
\hline Polarization & $>99 \%$ \\
\hline Pulse Length & $\sim 120 \mu \mathrm{s}$ \\
\hline Repetition Frequency & $500-1000 \mathrm{~Hz}$ adjustable \\
\hline
\end{tabular}

The sodium column density test lidar was set up on the other room beside the optical bench. A $300 \mathrm{~mm}$ telescope is used as the receiving telescope for the lidar. Main parameters of the lidar laser are listed in Table 2.

Table 2: Main parameters of the sodium column density lidar laser

\begin{tabular}{|l|l|}
\hline Linewidth & $<1.5 \mathrm{GHz}$ \\
\hline Repetition frequency & $20 \mathrm{~Hz}$ \\
\hline Single pulse energy & $\sim 50 \mathrm{~mJ}$ \\
\hline Pulse length & $\sim 9 \mathrm{~ns}$ \\
\hline
\end{tabular}

Measurement of the column density of the sodium atoms has been hold on the last two month of Year 2013 by University of Science and Technology of China and Wuhan Institute of Physics and Mathematics, CAS, while doing the photon return test of the sodium laser. The column density varied from $1.6 \times 10^{9} \mathrm{~cm}^{-2}$ to $2.8 \times 10^{9} \mathrm{~cm}^{-2}$ and average centroid height changed from $91 \mathrm{~km}$ to $96 \mathrm{~km}$. The average column density was $2.0 \times 10^{9} \mathrm{~cm}^{-2}$ during the test, as showed in Figure 2, which is lower than the measurement result in 2012 [9]. 

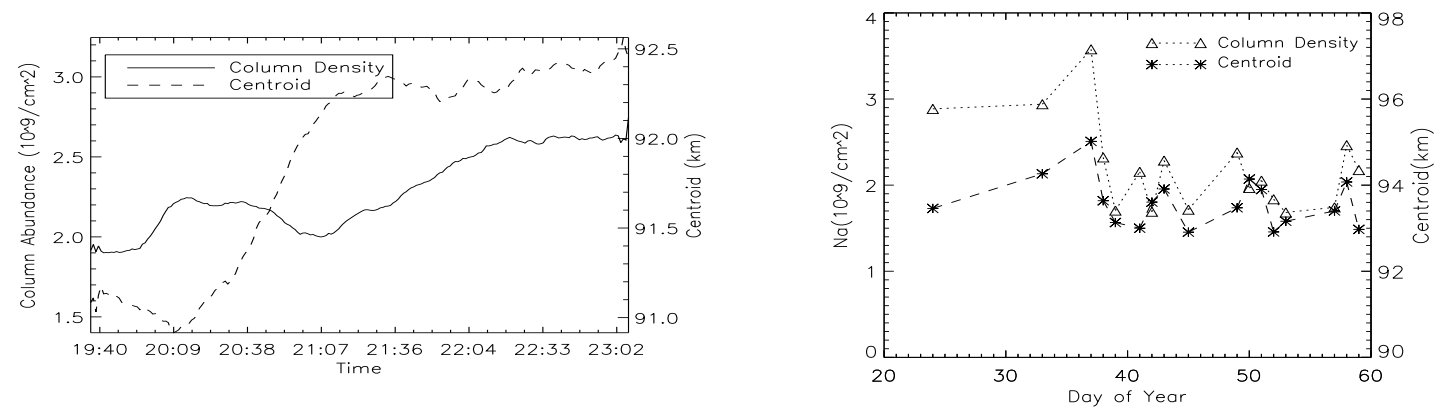

Figure 2: Column density profile of the sodium atoms measured by the lidar. Left: typical one night's result on November $12^{\text {th }}, 2013$ (in UTC time, local time should +8 hour). Right: all the measurement in the last two month of Year 2013.

Also, an atmospheric coherence length measurement equipment made by Anhui Institute of Optics and Fine Mechanics, CAS was set beside the 1.8 meter telescope. Continuous measurement of the atmospheric coherence length was held in the last two month of Year 2013. Figure 3 shows the results of the atmospheric coherence length. Good seeing occurred at about 20:00, 00:00, and 05:00 in local time in the evening of November $12^{\text {th }}$. Average atmospheric coherence length varied from $4 \mathrm{~cm}$ to $12 \mathrm{~cm}$ during the test.
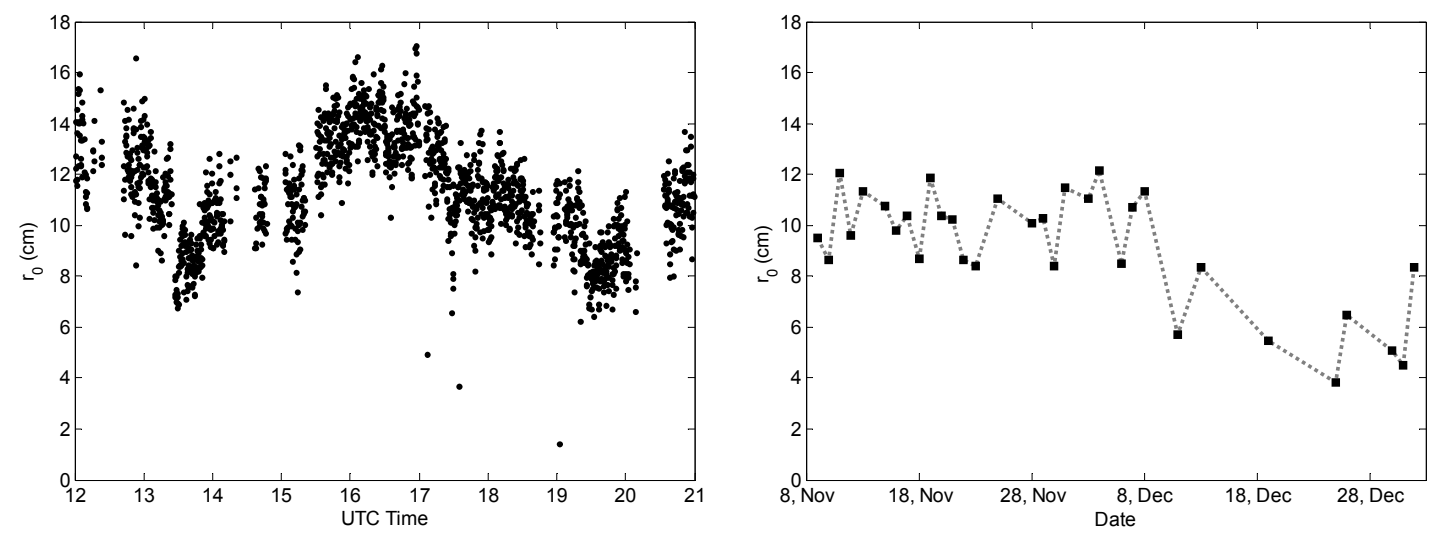

Figure 3: Results of atmospheric coherence length. Left: one night result on November $12^{\text {th }}, 2013$. Right: average of the atmospheric coherence length during one night's test.

\subsection{Laser Launch Telescope}

A $300 \mathrm{~mm}$ laser launch telescope (LLT) was built for the on-sky test. The $300 \mathrm{~mm}$ has the ability to launch the laser or be used as an imaging system for the stars. Laser diagnosis system, including measurements of laser power, beam quality, and polarization, was built in the $300 \mathrm{~mm}$ LLT. With a tip-tilt mirror, the LLT can do the real time control of the optical axes.

The basic structure of the $300 \mathrm{~mm}$ LLT is showed in Figure 4. The $300 \mathrm{~mm}$ LLT consists of three sub-systems: beam expander system, optical relay system, and imaging system. Designed parameters of the $300 \mathrm{~mm}$ LLT are listed in Table 3. Three remote-control flexure states are used to realize each designed function of the LLT.

Figure 5 show the test result of the 300mm LLT. An RMS of 0.048 wavelength was achieved for the test. The image quality of the $300 \mathrm{~mm}$ LLT is hard to test at a beam diameter of $240 \mathrm{~mm}$. The LGS spot image was used to estimate the imaging quality for the $300 \mathrm{~mm}$ LLT. This will be discussed in the next section. 
Table 3: Designed parameters of the 300mm LLT

\begin{tabular}{|l|l|}
\hline Launch location & Side Launch \\
\hline Transmission & $>92 \%$ \\
\hline Focal length & $90 \sim 210 \mathrm{~km}$ \\
\hline Beam quality & $\mathrm{SR}>0.98$ \\
\hline Field of view & \pm 2.5 \\
\hline Diameter of the primary mirror & $300 \mathrm{~mm}$ \\
\hline Diameter of the laser beam & $240 \mathrm{~mm} @ 1 / \mathrm{e}^{2}$ \\
\hline Polarization & $>99 \%$ \\
\hline Pointing precision & $<0.2 ” \mathrm{RMS}$ \\
\hline
\end{tabular}

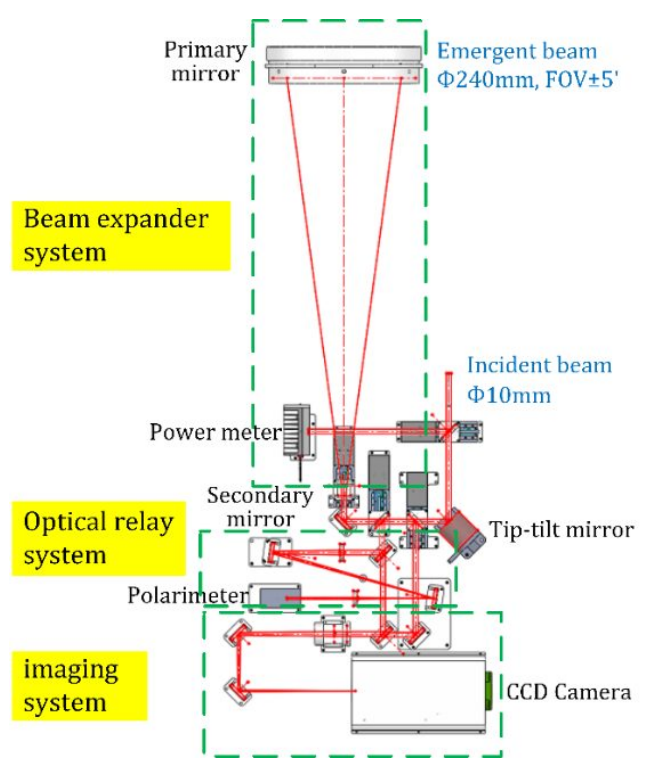

Figure 4: Schematic of the 300mm LLT structure. Green regions show 3 sub-systems respectively: beam expander system, optical relay system, and imaging system.
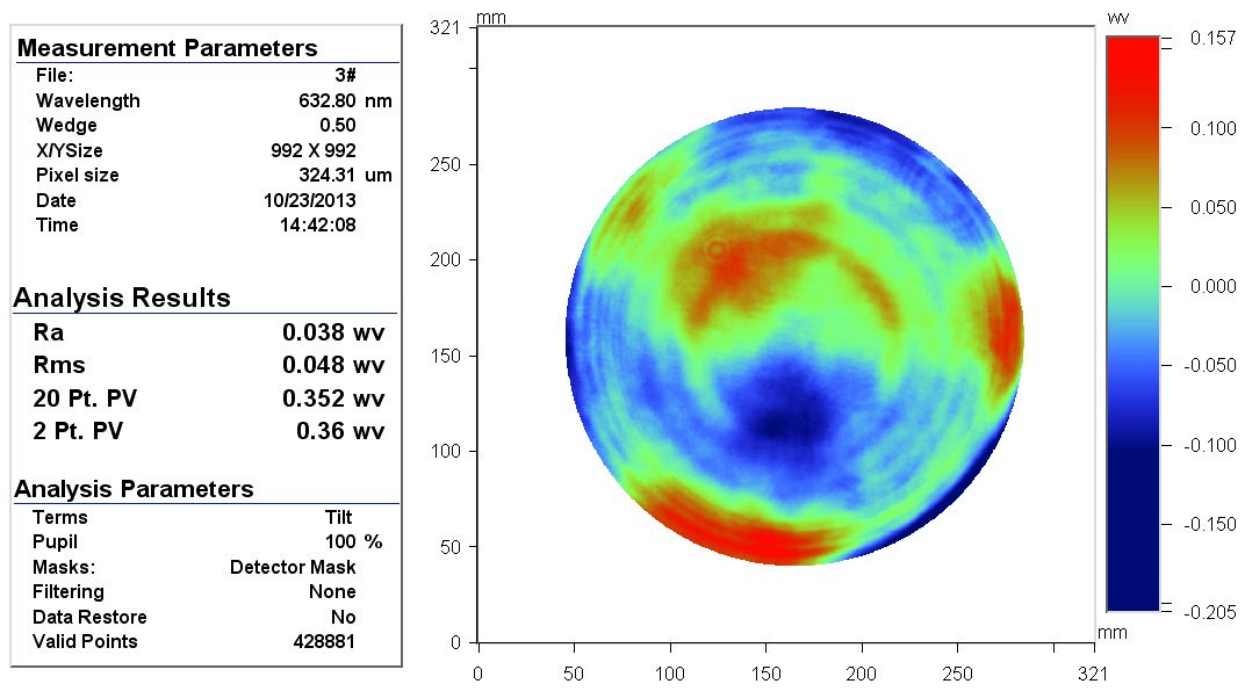

Figure 5: Wavefront test result of the $300 \mathrm{~mm}$ LLT. The test diameter of the LLT is $250 \mathrm{~mm}$ 


\subsection{Observations of the Sodium Beacon}

Usually there are three observation modes during a testing night:

The first and most common observation mode is using the CCD camera on the Nasmyth focus of the 1.8 meter telescope. This mode is used for measuring the magnitude and the photon return of the sodium beacon. A astronomical V-band filter (Andover KRON-V-50) is installed before the Nasmyth CCD camera. The transmission of the V-band filter has some difference from a standard Johnson-Cousin V-band filter, a post-processing method is used to eliminate the difference.

The second mode is using a high-speed CCD camera (Photometrics Cascade 128+) on a Coude focus. This mode is used for measuring the spot size of shot time scale of the sodium beacon. The total distance from the laser output position to the LLT is about $7 \mathrm{~m}$ and it is windy outside the 10000 cleaning room. This bring a jitter problem for the laser beam. It is hard to measure the spot size with a long exposure time using the Nasmyth CCD ( $>500 \mathrm{~ms})$. The sampling frequency for the high-speed CCD camera can be as fast as $400 \mathrm{~Hz}$. That make us possible to tune the LLT and the beam transfer optics (BTO) to get a best shape of the sodium beacon.

The last mode is using the PMT detector for the sodium column density lidar. The controlling software for the PMT is originally written for the lidar laser not for the sodium laser. A quantitative measurement for the photon returns of the sodium beacon cannot be get if using the PMT detector. However, it is still possible for us to get a qualitative result. Using the PMT we can optimize the wavelength and polarization of the sodium laser and get a maximum photon returns of the sodium beacon.

\section{ON-SKY TEST RESULTS AND DISCUSSION}

\subsection{Optimization of the Sodium Beacon: Spot Size and Brightness}

Sodium beacon spot size and shape are tuned into best condition before doing the photon return measurements. Using the short exposure time image from the second observation mode, the beam transfer optics and the LLT are tuned to make the sodium beacon spot as small as possible. Retuning of the spot size had been done with the old 200mm LLT after 2011 test and best images could reach spot size of 5-6 arcsec at shot time exposure $(<100 \mathrm{~ms})$. Distance between the LLT and 1.8 meter receiving telescope is $5.2 \mathrm{~m}$, corresponding to a separation of about 20 arcsec between the Rayleigh and sodium resonant scatting. Because the $300 \mathrm{~mm}$ LLT did not catch up with the schedule of the photon return test, most of the tests were held with the $200 \mathrm{~mm}$ LLT.
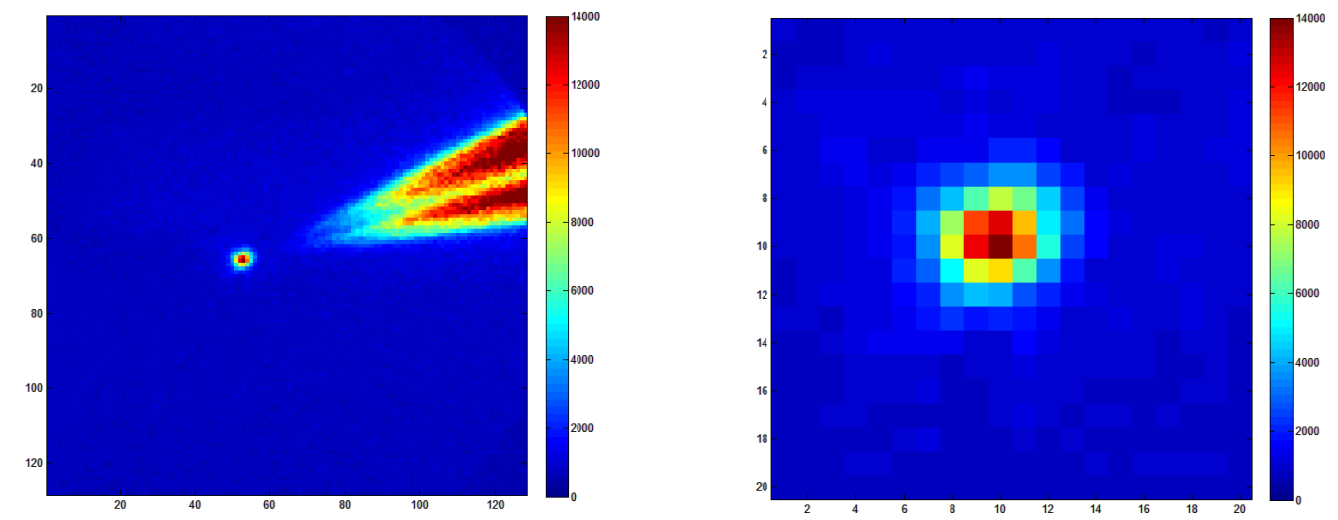

Figure 6: Typical high speed CCD image of a sodium beacon with the 200mm LLT (exposure time: 20ms, unit of image: arcsec). The field of view of the CCD camera is $2.1 \times 2.1 \mathrm{arcmin}$.

LGS spot size with the $300 \mathrm{~mm}$ LLT was also measured. After the photon return test, the $300 \mathrm{~mm}$ LLT was mounted on the side of 1.8 meter telescope to test the imaging quality of the LLT. With the new $300 \mathrm{~mm}$ LLT, the best spot size of the sodium beacon is about 3.5 arcsec FWHM, as showed in Figure 7. 


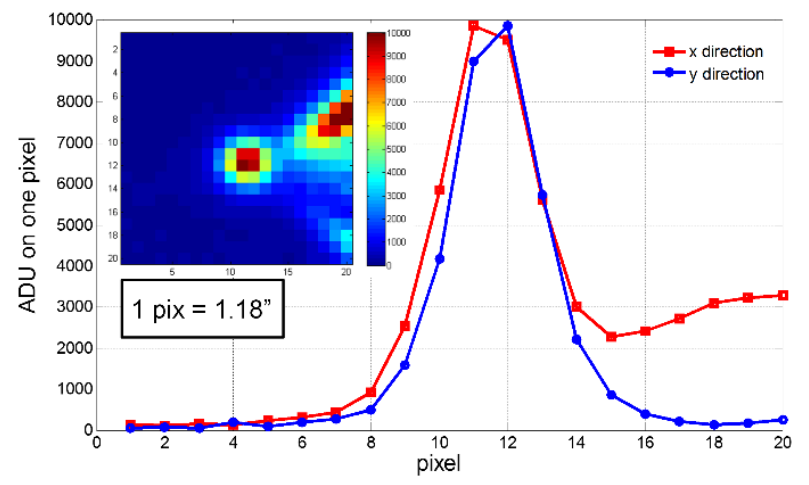

Figure 7: LGS spot size with the 300mm LLT (exposure time 500ms)

The center wavelength of the laser has to be tuned exactly the same as the center of the sodium $\mathrm{D}_{2} \mathrm{a}$ line if a bright sodium beacon want to be generated. To find the best tune of the laser, a series of images at different laser center wavelength were taken.

A $\lambda / 4$ plate was put in the BTO to convert the linear polarized light to circular polarized light. Measurement of the ADU of the sodium beacon at different rotate angle of the $\lambda / 4$ plate was accomplished to find the right rotate angle of the $\lambda / 4$ plate. Using the CCD camera on the Nasmyth focal plane from the first observation mode and using the PMT detector of the sodium column density lidar from the third observation mode get the same conclusion of the $\lambda / 4$ plate rotate angle. Result of the measurement using the CCD camera is showed in Figure 8.

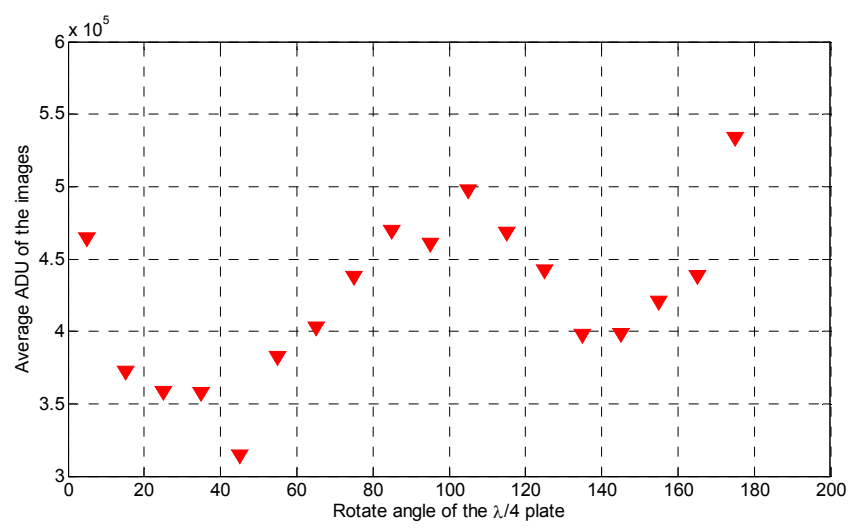

Figure 8: Result of the measurement of the sodium beacon ADU at different rotate angle of the $\lambda / 4$ plate. The circular polarized light comes out when the rotate angle of the $\lambda / 4$ plate is $95^{\circ}$, which is the same as the result from the measurement of the PMT detector.

\subsection{Photon Return Test Results}

Because the laser output power is not very stable at the maximum power level, usually the measurements of the brightness of the sodium beacon were tested in $15-20 \mathrm{~W}$ output level, at repetition frequency of $500 \mathrm{~Hz}$. With the astronomical V-band filter, nature star images were also taken to calibrate the magnitude of the sodium beacon. Data reductions of the stars with their spectral types, quantum efficiency of the Nasmyth CCD camera, and transmission of the V-band filter are made in order to calculate the absolute magnitude of the sodium beacon..

The brightest beacon we got at $500 \mathrm{~Hz}$ RPF is $8.2 \mathrm{Mag}$ in V-band while using a circular polarized light and the output power of the laser is $19 \mathrm{~W}$ at November $16^{\text {th }}, 2013$. Also difference between circular and linear polarized light have been tested in that day. Figure 9 and Figure 10 show the difference between two kinds of polarized light: about $60 \%$ more photons will be get if the circular polarized light is used. 


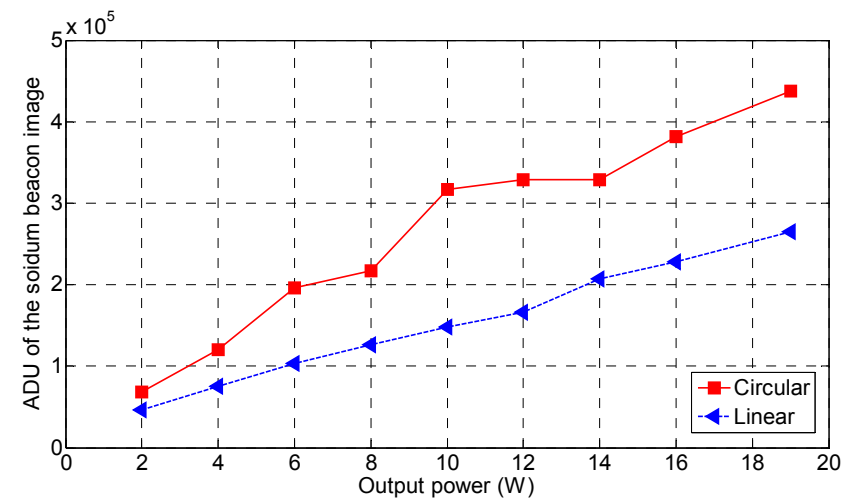

Figure 9: Difference between the ADU of the sodium beacon image at various laser power lever when using a circular or linear polarized light. The augmentation of circular light in $10 \mathrm{~W}$ and $12 \mathrm{~W}$ level may cause by the boost of the sodium layer.

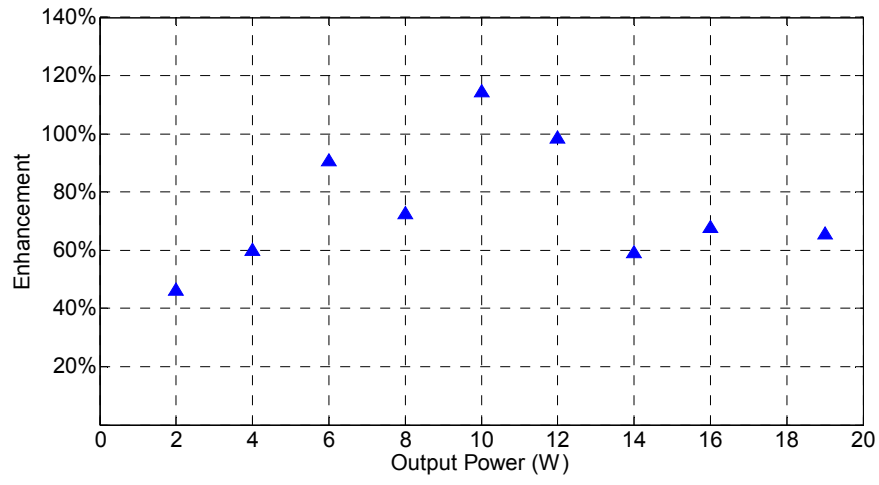

Figure 10: Enhancement of the photon return if a circular polarized light is used.

The definition of coupling efficiency comes from Eqs.(7) in Holzlöhner's paper [11].

$$
S_{c e}=\frac{\Phi L^{2}}{P\left(T_{a}\right)^{2 X} C_{\mathrm{Na}} X}
$$

Where $\Phi$ is the photon flux on the detector (unit photons $/ \mathrm{s} / \mathrm{m} 2), L$ is the vertical distance from the receiving telescope to the sodium layer, $P$ is the laser power after the LLT, $T_{a}$ is the atmos-phere transmission, $X=1 /(\sec (\theta))$ and $\theta$ is the laser launch zenith angle, $C_{\mathrm{Na}}$ is the column density of the sodium atoms.

Coupling efficiency can be as much as 100 photons $/ \mathrm{s} / \mathrm{W} /($ atoms $/ \mathrm{m} 2)$ at $19 \mathrm{~W}$ output power for a linear polarized light. This could have an enhancement and increase to $160 \mathrm{photons} / \mathrm{s} / \mathrm{W} /($ atoms $/ \mathrm{m} 2)$ when using a circular polarized light. As output power drops down, the effect of the saturation of the sodium atoms will decrease, and the coupling efficiency will increase as showed in Figure 11.

Note that transmission from the laser machine to the outcome of the LLT cannot be measured, an estimation of 0.8 is used during the calculation of the coupling efficiency. The sodium column density lidar and the photon return test of the sodium beacon cannot be work at the same time. So the sodium column density we used in the calculation is an estimation based on measured sodium profile data. 


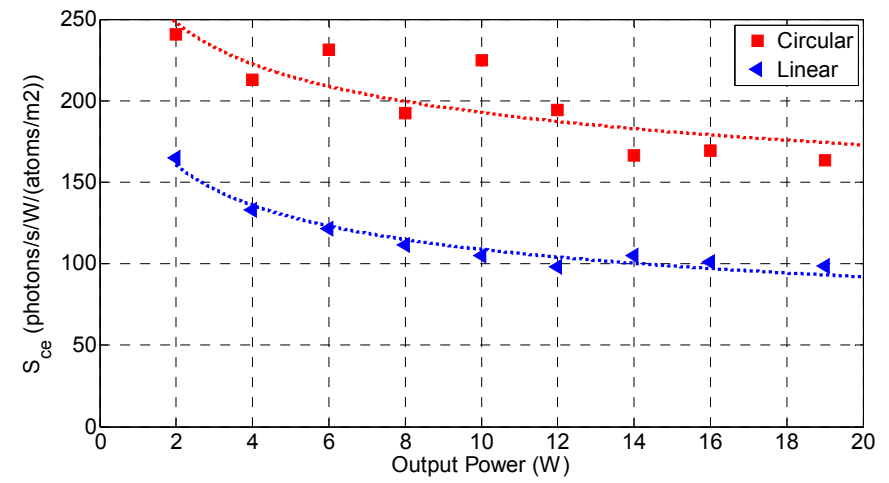

Figure 11: Coupling efficiency of the TIPC sodium laser at different power level.

\section{CONCLUSIONS}

Using the TIPC long pulsed sodium laser and the laser launch telescope, a sodium beacon at a spot size of 7 arcsec and a magnitude 8.2 in V-band was successfully observed. The coupling efficiency of the TIPC laser could reach 160 photons $/ \mathrm{s} / \mathrm{W} /($ atoms $/ \mathrm{m} 2)$ when using a circular polarized light or 100 photons $/ \mathrm{s} / \mathrm{W} /($ atoms $/ \mathrm{m} 2)$ when using a linear polarized light at current spot size. Sodium data profile is also presented and average column density of the sodium atoms was about $2.0 \times 10^{9} \mathrm{~cm}^{-2}$. Average atmospheric coherence length varied from $4 \mathrm{~cm}$ to $12 \mathrm{~cm}$ during the test nights.

\section{REFERENCE}

[1] Céline d'Orgeville, Mark R. Chun, Jacques Sebag, Corinne Boyer, et al., "Gemini Manna Kea Laser Guide Star System", Proc. SPIE 3762 (1999).

[2] Peter L. Wizinowich, David Le Mignant, Antonin H. Bouchez, et al., "The W. M. Keck Observatory Laser Guide Star Adaptive Optics System: Overview”, PASP, 118:297-309 (2006).

[3] D.Bonaccini Calia, E. Allaert, J. L. Alvarez, C. Araujo Hauck, et al., "First Light of the ESO Laser Guide Star Facility", Proc. SPIE 6272, 627207 (2006).

[4] Yutaka Hayano, Yoshihiko Saito, Meguru Ito, et al., "The laser guide star facility for Subaru Telescope", Proc. SPIE 6272, 627247 (2006).

[5] Richard Joyce, Corinne Boyer, Larry Daggert, et al., "The Laser Guide Star Facility for the Thirty Meter Telescope", Proc SPIE 6272, 62721H (2006).

[6] Ian Lee, Munib Jalali, Neil Vanasse, et al., "20W and 50W Guidestar Laser System Update for the Keck I and Gemini South Telescopes", Proc SPIE 7015, 70150N (2008).

[7] S. Rabien, N. Ageorges, R. Angel, et al., "The Laser Guide Star Program for the LBT”, Proc. SPIE 7015, 701515 (2008).

[8] J. L. Alvarez, J. Beltran, I Munoz, G. Valdes, et al., "Laser Guide Star Facility at La Silla Paranal Observatory", Proc. SPIE 7736, 77364X (2010).

[9] Kai Wei, Yong Bo, Xianghui Xue, et al., "Photon returns test of the pulsed sodium guide star laser on the 1.8 meter telescope", Proc. SPIE 8447, 84471R (2012).

[10] S. M. Rochester, A. Otarola, C. Boyer, et al., "Modeling of pulsed laser guide stars for the Thirty Meter Telescope project", JOSA B, 29: 2176-2188 (2012).

[11] R. Holzlöhner, S. M. Rochester, D. Bonaccini Calia, et al., "Optimization of cw sodium laser guide star efficiency", A\&A 510, A20 (2010) 